\title{
PLS for V2I Communications Using Friendly Jammer and Double kappa-mu Shadowed Fading
}

\author{
Neji Mensi and Danda B. Rawat \\ Department of Electrical Engineering and Computer Science \\ Howard University \\ Washington, DC 20059, USA \\ neji.mensi@bison.howard.edu,danda.rawat@howard.edu
}

\author{
Elyes Balti \\ Wireless Networking and Communications Group \\ The University of Texas at Austin \\ Austin, TX 78712, USA \\ ebalti@utexas.edu
}

\begin{abstract}
The concept of intelligent transportation systems (ITS) is considered to be a highly promising area of research due to its diversity of unique features. It is based mainly on the wireless vehicular network (WVN), where vehicles can perform sophisticated services such as sharing real-time safety information. To ensure high-quality service, WVN needs to solve the security challenges like eavesdropping, where malicious entities try to intercept the confidential transmitted signal. In this paper, we are going to provide a security scheme under the Double kappa-mu Shadowed fading. Our solution is based on the use of a friendly jammer that will transmit an artificial noise (AN) to jam the attacker's link and decrease its eavesdropping performances. To evaluate the efficiency of our solution, we investigated the outage probability for two special cases: Nakagami-m and Rician shadowed while taking into consideration the density of the blockage and the shadowing effects. We also studied the average secrecy capacity via deriving closed-form expressions of the ergodic capacity at the legitimate receiver and the attacker for the special case: Nakagami-m fading distribution.

Index Terms-Physical Layer Security, Eavesdropping, Wireless Vehicular Network, Artificial Noise, Double $\kappa-\mu$ Shadowed Fading.
\end{abstract}

\section{INTRODUCTION}

\section{A. Background and Literature Review}

Wireless vehicular network (WVN) has been exponentially evolving by taking advantage of sophisticated technologies such as artificial intelligence (AI) [1], machine learning [2], Millimeter Wave (mmWave) [3]-[7], and 5G [8]-[10], etc. It offers a variety of advanced features like real-time alerting messages [11], cloud services [12], [13], etc. However, as any communication network, WVN is subject to many challenges, especially the security issue which is a peer parameter that guarantees a certain acceptable quality of services (QoS).

Several research papers discussed the security challenges and proposed a variety of solutions from different OSI (Open Systems Interconnection model) layers perspective [14], [15]. Regarding the physical layer security (PLS), it has been proven that securing the communication at this level is an efficient scheme to deal with passive threats like the eavesdropping attacks. In this kind of menace, the malicious network entity is able to listen to a private communication by intercepting the transmitted signal and revealing the confidential information.

This work was supported in part by the U.S. National Science Foundation (NSF) under the grant CNS-1650831.
What makes the eavesdropping attacks very critical is the fact that it is difficult to be detected by the victims since it could be processed without leaving any traces. To deal with this security dilemma, some related works proposed the use of artificial noise (AN) where the transmitter perturbs the attacker channel by sending a dedicated signal [16], [17]. This signal is generated orthogonally to the main link between the legitimate entities so that it will affect only the eavesdropper link. Other papers studied the employment of a friendly jammer $(\mathrm{J})$ which has the responsibility of jamming the eavesdroppers' channels instead of the transmitter [18], [19].

In general, by employing a jammer $J$, as a third network entity, could be more efficient in the case of multiple eavesdroppers attacks. In other words, if the network is subject to several attacks, it is better to have a jammer node to deal with all the attackers rather than each communicating node deals with all the attackers by itself (it will be very expensive in terms of power if each transmitter will dedicate a fraction of its power to jam all the eavesdroppers' channels).

\section{B. Our Contribution}

In our paper, we are focusing on using the friendly jammer to protect V2I communications. As a channel fading, we adopt the Double Shadowed $\kappa-\mu$ Fading Model, noted by $\mathcal{D}(\cdot)$, recently presented in [20], which is more general and covers wide ranges of fading such as double shadowed Rice, Rician shadowed, Nakagami-q, Nakagami-m, Rayleigh, onesided Gaussian, etc. We highlight our contributions as follows:

- We propose the use of friendly jammer $J$ in V2I communications under an eavesdropping attack scenario.

- We adopt the new channel model $\mathcal{D}(\cdot)$.

- We derive a closed form expression for the ergodic capacity at the receiver under $\mathcal{D}(\cdot)$ model.

- We derive a closed form expression for cumulative distribution function (CDF) of the signal-to-interferenceplus-noise-ratio (SINR) and the ergodic capacity at the eavesdropper while considering Nakagami-m as special case of $\mathcal{D}(\cdot)$.

- We examine the impact of the blockage density at the receiver by adopting the special case model Rician shadowed. 


\section{Paper Structure}

The paper is constructed as follows: Section II introduces the system model. Section III studies the outage probability while Section IV examines the ergodic capacity and the secrecy capacity. Then, Section V evaluates the performance of the security approach based on numerical results. Finally, we outline our conclusion in Section VI.

\section{SYSTEM MODEL}

\section{A. Vehicular Communications and Attack Model}

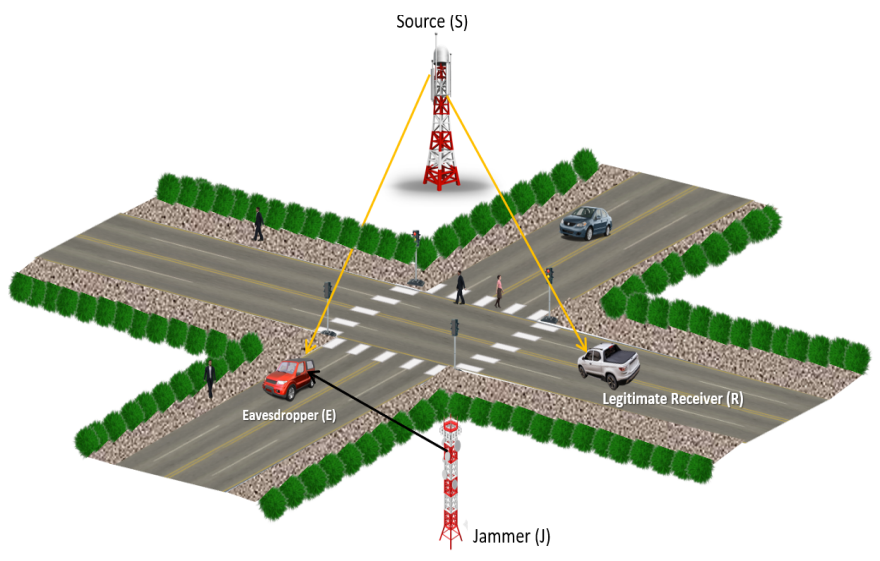

Fig. 1: V2I communications in the presence of multiple eavesdroppers.

In V2I communications, base stations (BSs) and vehicles should be able to exchange information and data securely. However, the transmitted signals may be subject to an intended overhearing where an eavesdropper intercepts the signal and reveals the secret messages. In Fig. 1, we have a typical model where the attacker $E$ is listening to the communication between the source $S$ (the base station) and the legitimate receiver $R$. In this case, $J$ tends to protect the network by sending AN to the attacker $E$, while $R$ is immune since the AN is orthogonal to its channel.

\section{B. Channel Model}

The received signal at $R$ and $E$ are respectively:

$$
\begin{aligned}
y_{R} & =\sum_{n=1}^{N} h_{S, R ; n} x_{I}+\sum_{k=1}^{K} h_{J, R ; k} x_{J}+w_{R} \\
& =\sum_{n=1}^{N} h_{S, R ; n} x_{I}+w_{R}, \\
y_{E} & =\sum_{n=1}^{N} h_{S, E ; n} x_{I}+\sum_{k=1}^{K} h_{J, E ; k} x_{J}+w_{E},
\end{aligned}
$$

where the channel model parameters are defined in Table I.

As we can deduce from Eq. (1), $\sum_{k=1}^{K} h_{J, R} x_{J}=0$, which means that the jamming signal will only affect the attacker while conserving the same signal at the legitimate receiver. The received SNR and SINR at $R$ and $E$, respectively, are

$$
\gamma_{R}=\frac{\sum_{n=1}^{N} P_{S}\left|g_{S, R}\right|^{2} r_{S, R ; n}^{-\delta}}{\sigma_{w_{R}}^{2}}=\sum_{n=1}^{N} \gamma_{R ; n},
$$

\begin{tabular}{|c|c|}
\hline Parameters & Description \\
\hline $\mathrm{N}$ & Number of antennas at the BS \\
\hline $\mathrm{K}$ & Number of antennas at the Jammer $J$ \\
\hline$h_{a, b ; c}$ & $\begin{array}{l}\text { The fading amplitude of the channel corresponding } \\
\text { to the link between the antenna } c \text { of the node } a \\
\text { and the receiving node } b \text {. } \\
\qquad a \in\{J, S\} \text { and } b \in\{R, E\}\end{array}$ \\
\hline & $g_{a, b}$ is the channel gain where $\left|g_{a, b}\right|^{2} \sim \mathcal{D}(\cdot)$ \\
\hline $\begin{aligned} & h_{a, b ; c} \\
= & g_{a, b ; c} \sqrt{r_{a, b ; c}^{-\delta}}\end{aligned}$ & $\begin{array}{l}r_{a, b ; c} \text { is the distance between the antenna } c \\
\text { of the node } a \text { and the receiving node } b \\
\delta \text { is path loss exponent }\end{array}$ \\
\hline$x_{I}$ & $\begin{array}{l}\text { The confidential information signal } \\
\text { sent by } S \text { with power } P_{S} / \text { antenna }\end{array}$ \\
\hline$x_{J}$ & $\begin{array}{l}\text { jamming signal (AN) emitted by } J \\
\text { with power } P_{J} \text { /antenna }\end{array}$ \\
\hline$w_{b}$ & $\begin{array}{l}\text { The additive white Gaussian noise (AWGN) } \\
\text { at the node } b \text { with variance } \sigma_{w_{b}}^{2}\end{array}$ \\
\hline \multicolumn{2}{|c|}{$\sum_{n=1}^{N} P_{S}\left|h_{S, E \cdot n}\right|^{2}$} \\
\hline \multicolumn{2}{|c|}{$\gamma_{E}=\frac{\sum_{k=1}^{K} P_{J}\left|h_{J, E ; k}\right|^{2}+\sigma_{w_{E}}^{2}}{\sum_{N}}$} \\
\hline \multicolumn{2}{|c|}{$\overline{1+\sum_{k=1}^{K} \gamma_{J ; k}}=\overline{1+\gamma_{J}}$} \\
\hline
\end{tabular}

TABLE I: Channel model parameters description

where $\gamma_{R ; n} \sim \mathcal{D}(\cdot)$ and $\gamma_{I ; n} \sim \mathcal{D}(\cdot)$ are, respectively, the SNRs corresponding to the confidential signal received at $R$ and $E$ via the $n$-th antenna, while $\gamma_{J: k} \sim \mathcal{D}(\cdot)$ is the SNR due to the jamming signal sent by $J$ at $E$ via the $k$-th antenna. The general probability density function (PDF) and CDF of the random variable (RV) $\gamma_{l}$, where $l \in\{(I ; n),(J ; k),(R ; n)\}$, are respectively

$$
\begin{aligned}
& f_{\gamma_{l}}(\gamma)=\frac{\left(s_{l}-1\right)^{s_{l}} c_{l}^{c_{l}} T_{l}^{\mu_{l}} \gamma^{\mu_{l}-1} \bar{\gamma}^{s_{l}}}{\left(c_{l}+\mu_{l} \kappa_{l}\right)^{c_{l}} B\left(s_{l}, \mu_{l}\right)\left(T_{l} \gamma+\left(s_{l}-1\right) \bar{\gamma}\right)^{s_{l}+\mu_{l}}} \\
& \times_{2} F_{1}\left(c_{l}, s_{l}+\mu_{l} ; \mu_{l} ; \frac{K_{l} \mu_{l} \kappa_{l} \gamma}{T_{l} \gamma+\left(s_{l}-1\right) \bar{\gamma}}\right) \text {, } \\
& F_{\gamma_{l}}(\gamma)=\left(\frac{c_{l}}{c_{l}+\kappa_{l} \mu_{l}}\right)^{c_{l}}\left(\frac{T_{l} \gamma}{\bar{\gamma}\left(s_{l}-1\right)}\right)^{\mu_{l}} \sum_{i=0}^{\infty}\left(\frac{K_{l} \mu_{l} \kappa_{l} \gamma}{\left(s_{l}-1\right) \bar{\gamma}}\right)^{i} \\
& \frac{\left(c_{l}\right)_{i}+\left(i+\mu_{l}\right)_{s_{l}}}{i ! \Gamma\left(s_{l}\right)\left(i+\mu_{l}\right)}{ }_{2} F_{1}\left(i+\mu_{l}, i+\mu_{l}+s_{l} ; i+\mu_{l}+1 ; \mu_{l} ; \tau\right) \text {, }
\end{aligned}
$$

\begin{tabular}{|c|c|c|}
\hline SNR & Parameters & Description \\
\hline \multirow{6}{*}{$\gamma_{l}$} & $c_{l}$ & Shape of the Nakagami-m RV \\
\hline & $s_{l}$ & Shape of the inverse of Nakagami-m RV \\
\hline & $\mu_{l}$ & Number of multipath clusters \\
\hline & $\kappa_{l}$ & $\begin{array}{l}\text { The ratio of the total power of the dominant } \\
\text { components to the scattered waves }\end{array}$ \\
\hline & $T_{l}$ & $\mu_{l}\left(1+\kappa_{l}\right)$ \\
\hline & $K_{l}$ & $\frac{K_{l}}{\left(c_{l}+\mu_{l} * \kappa_{l}\right)}$ \\
\hline
\end{tabular}

where ${ }_{2} F_{1}(\cdot, \cdot ; \cdot ;$.$) represents the Hypergeometric function,$ $B(\cdot, \cdot)$ denotes the Beta function, $\tau=\frac{-T_{l} \gamma}{\bar{\gamma}\left(s_{l}-1\right)}$ and $(x)_{i}=$ $\frac{\Gamma(x+i)}{\Gamma(x)}$ is the the Pochhammer symbol. For the sake of organization, the parameters on which depends the distribution of $\gamma_{l}$, are presented in Table. II.

TABLE II: $\mathcal{D}(\cdot)$ parameters description [20] 


\section{Outage Probability Analysis}

\section{A. Outage Probability at the Legitimate Receiver}

To analyze the outage probability at $R$, we have to refer to the corresponding CDF. We know that $\gamma_{R ; n} \sim \mathcal{D}(\cdot)$, hence its $\mathrm{CDF}$ is given by Eq. (6). However, the closed-form expression of the CDF corresponding of $\sum_{n=1}^{N} \gamma_{R ; n}$ is not tractable. Therefore, we are assuming that $\mathrm{N}=1$, which make the outage probability of $\gamma_{R}$ represented by Eq. (6). At this stage, we are referring to the special fading case Rician shadowed under the following substitutions: $c_{R} \rightarrow \infty$ and $\mu=1$ [20]. Therefore, the CDF is expressed as follows [21]

$F_{\gamma_{R}}(\gamma)=\frac{1}{\Gamma(m)}\left(\frac{m}{\Xi}\right)^{m} \sum_{i=0}^{\infty} \frac{\Gamma(m+i) \gamma\left(i+1, \frac{\gamma}{\bar{\gamma} 2 \sigma^{2}}\right)}{\sigma^{2 i} 2^{i} i ! \Gamma(1+i)\left(\frac{1}{2 \sigma^{2}}+\frac{m}{\Xi}\right)^{m+i}}$,

where $\gamma(\cdot, \cdot)$ is the Incomplete Gamma function, $\Xi$ is the average power of the line of sight (LOS) component, $2 \sigma^{2}$ is the average power of the scatter component, $m$ is fading figure which represents the fading severity. Therefore, the outage related to the density of shadowing, $F_{\gamma_{S D}}$ could be presented by

$$
F_{\gamma_{S D}}(\gamma)=p_{l o s} F_{\gamma_{S D}}^{\text {los }}(\gamma)+\left(1-p_{\text {los }}\right) F_{\gamma_{S D}}^{\text {nlos }}(\gamma)
$$

where $F_{\gamma_{S D}}^{\text {los }}(\gamma)$ and $F_{\gamma_{S P}}^{\text {nlos }}(\gamma)$ are the CDFs of the SNR evaluated when the link is LOS, and non-line-of-side (NLOS), respectively.

\section{B. Outage Probability at the Attacker}

It is complex to derive a closed-form expression of the CDF at the eavesdropper $E$. However, we can take advantage of the $\mathcal{D}(\cdot)$ generality by considering special channel model cases by manipulating its parameters. We suggest that $h_{S, E}$ and $h_{J, E}$ follow Nakagami-m distribution by fixing $c_{R}=s_{R}=\infty$, $\mu_{I}=1$ and $\kappa_{I}=m$ [20]. Hence, $\gamma_{I ; n}$ and $\gamma_{J ; k}$ (see Eq. (4) ) are described by Gamma distribution $\left(\gamma_{I ; n} \sim G\left(\nu_{I, n}, \beta_{I, n}\right)\right.$ and $\left.\gamma_{J: k} \sim G\left(\nu_{J, k}, \beta_{J, k}\right)\right)$, which has the following PDF and $\mathrm{CDF}$

$$
\begin{gathered}
f_{\gamma_{d}}(\gamma)=\frac{\beta_{d}^{\nu_{d}} \gamma^{\left(\nu_{d}-1\right)} \exp \left(-\beta_{d} \gamma\right)}{\Gamma\left(\nu_{d}\right)}, \\
F_{\gamma_{d}}(\gamma)=1-\frac{\Gamma\left(\nu_{d}, \beta_{d} \gamma\right)}{\Gamma\left(\nu_{d}\right)},
\end{gathered}
$$

where $d \in\{(I ; n),(J ; k)\}, \beta_{h}$ and $\nu_{h}$ are, respectively, the scale and the shape parameters. Each antenna of the BS has the same distance from the attacker because the antennas are collocated. Therefore the parameter $\beta_{I, n}$ is the same for all antennas $n \in 1, \ldots, N\left(\beta_{I}=\beta_{I ; n}\right)$ since $\beta_{S, E ; n}=\frac{P_{S} r_{S, E ; n}^{-\delta}}{\sigma_{w_{E}}^{2}}$. We assume that all $N$ links have the same scale parameter, which means $\nu_{I}=\sum_{n=1}^{N} \nu_{I ; n}$. Therefore $\sum_{n=1}^{N} \gamma_{I ; n}=\gamma_{I} \sim$ $G\left(\nu_{I}, \beta_{I}\right)$. The same analogy applied to the signal issued by the jammer where $\sum_{k=1}^{K} \gamma_{J ; k}=\gamma_{J} \sim G\left(\nu_{J}, \beta_{J}\right)$. For the sake of mathematical simplicity, we assume that $\nu_{h}$ is a positive integer. Accordingly, the $\mathrm{CDF}$ has the subsequent series expansion as:

$$
F_{\gamma_{d}}(\gamma)=1-\sum_{n=0}^{\nu_{d}-1} \frac{\left(\beta_{d} \gamma\right)^{n} e^{-\beta_{d} \gamma}}{n !}
$$

Before deriving the $\mathrm{CDF}$ at $E$ following the aforementioned special cases, we should mention that we verified and proved obtaining Nakagami-m distribution by using the general PDF of the envelope corresponding to $\mathcal{D}(\cdot)$ and substituting the appropriate parameters (Please refer to the Appendix).

By referring to Eq. (4), Eq. (9), and Eq. (11), the CDF at the attacker can be derived using the following expression

$$
\begin{aligned}
& F_{\gamma_{E}}=\int_{0}^{\infty} F_{\gamma_{I}}\left(\gamma\left[1+\gamma_{J}\right]\right) f_{\gamma_{J}}\left(\gamma_{J}\right) d \gamma_{J} \\
& =1-\frac{e^{-\beta_{I} \gamma_{\beta_{J}} \nu_{J}}}{\Gamma\left(\nu_{J}\right)} \sum_{n=0}^{\nu_{I}-1} \sum_{q=0}^{i}\left(\begin{array}{l}
n \\
q
\end{array}\right) \int_{0}^{\infty} \frac{\gamma_{J}^{\Omega-1} e^{-\gamma_{J}\left(\beta_{I} \gamma+\beta_{J}\right)}}{n !\left(\beta_{I} \gamma\right)^{-n}} d \gamma_{J}
\end{aligned}
$$

where $\Omega=q+\nu_{J}$.

Then, by referring to [22, Eq (3.351.3)], we obtain

$F_{\gamma_{E}}(\gamma)=1-\frac{e^{-\beta_{I} \gamma_{\beta_{J}}{ }^{\nu_{J}}}}{\Gamma\left(\nu_{J}\right)} \sum_{n=0}^{\nu_{I}-1} \sum_{q=0}^{i}\left(\begin{array}{l}n \\ q\end{array}\right) \frac{\Gamma(\Omega)\left(\beta_{I} \gamma+\beta_{J}\right)^{-\Omega}}{n !\left(\beta_{I} \gamma\right)^{-n}}$

\section{Average Secrecy Capacity Analysis}

The generalized formula of the ergodic capacity for a given SNR $\gamma$ is expressed by

$$
\begin{aligned}
\bar{C}_{p} & =\mathbb{E}\left[\log _{2}(1+\gamma)\right]=\int_{0}^{\infty} \log _{2}(1+\gamma) f_{\gamma_{p}}(\gamma) d \gamma \\
& =\frac{1}{\log (2)} \int_{0}^{\infty} \frac{\bar{F}_{\gamma_{p}}(\gamma)}{1+\gamma} d \gamma,
\end{aligned}
$$

where $p \in\{L, E\}$ and $\bar{F}_{\gamma_{p}}$ is the complementary of the CDF. The general average secrecy capacity $\bar{C}_{S}$ can be defined by

$$
\bar{C}_{s}= \begin{cases}\bar{C}_{R}-\bar{C}_{E}, & \text { if } \gamma_{R}>\gamma_{E} \\ 0, & \text { if } \gamma_{R}<\gamma_{E}\end{cases}
$$

where $\bar{C}_{L}$ is the average capacity of the main link (between $S$ and $R$ ) and $\bar{C}_{E}$ is the average capacity at the eavesdropper $E$.

\section{A. Ergodic Capacity at the Legitimate Receiver}

By referring to Eqs. (5, 6, and 14), the ergodic capacity at the legitimate receiver can be expressed by:

$$
\begin{aligned}
\bar{C}_{R}= & \int_{0}^{\infty} \frac{\left[\left(s_{R}-1\right) \bar{\gamma}_{L}\right]_{R} T_{R}^{\mu_{R}}}{\log (2) B\left(s_{R}, \mu_{R}\right)}\left(\frac{c_{R}}{\left.c_{R}+\mu_{R} \kappa_{I}\right)}\right)^{c_{R}} \\
& \times \sum_{i=0}^{\infty} \frac{\left(c_{R}\right)_{i}\left(s_{R}+\mu_{R}\right)_{i}\left(K_{R} \mu_{R} \kappa_{I}\right)^{i}}{i !\left(\mu_{R}\right)_{i}} \\
& \times \frac{\log (1+\gamma) \gamma_{R}^{\mu_{R}+i-1}}{K \gamma+\left[\left(s_{R}-1\right) \bar{\gamma}_{L}\right]^{i+s_{R}+\mu_{R}}} d \gamma
\end{aligned}
$$


To find a closed form of the aforementioned expression, we rewrite the following expressions as follow [23]

$$
\begin{gathered}
\frac{1}{(T \gamma+\Phi)^{\eta}}=\frac{1}{\Phi^{\eta} \Gamma(\eta)} G_{1,1}^{1,1}\left(\frac{T}{\Phi} \mid \begin{array}{c}
1-\eta \\
0
\end{array}\right) \\
\log (1+\gamma)=G_{2,2}^{1,2}\left(\gamma \mid \begin{array}{c}
1,1 \\
1,0
\end{array}\right),
\end{gathered}
$$

where $G_{p, q}^{m, n}(-\mid(\cdot, \cdot))$ is the Meijer G-Function, $\Phi=\left(s_{R}-\right.$ 1) $\bar{\gamma}_{L}, \alpha=\mu_{R}+i$, and $\eta=i+s_{R}+\mu_{R}$.

Then, by substituting Eq. (17) and Eq. (18) in Eq. (16) and by referring to the $[24,07.34 .21 .0011 .01]$, we obtain:

$$
\begin{aligned}
& \bar{C}_{R}=\frac{\left[\left(s_{R}-1\right) \bar{\gamma}_{L}\right]_{R} T_{R}^{\mu_{R}}}{\log (2) B\left(s_{R}, \mu_{R}\right)}\left(\frac{c_{R}}{\left.c_{R}+\mu_{R} \kappa_{I}\right)}\right)^{c_{R}} \\
& \times \sum_{i=0}^{\infty} \frac{\left(c_{R}\right)_{i}\left(s_{R}+\mu_{R}\right)_{i}\left(K_{R} \mu_{R} \kappa_{I}\right)^{i}}{i !\left(\mu_{R}\right)_{i}}
\end{aligned}
$$

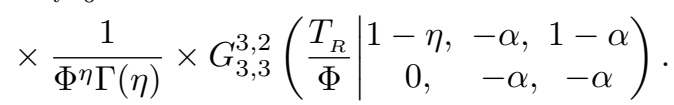

\section{B. Ergodic Capacity at the Eavesdropper}

Using Eq. (13) and Eq. (14), we can write the ergodic capacity as follows

$$
\bar{C}_{E}=\frac{\beta_{J}^{\alpha_{J}}}{\Gamma\left(\alpha_{J}\right)} \sum_{n=0}^{\nu-1} \sum_{q=0}^{n}\left(\begin{array}{l}
n \\
q
\end{array}\right) \int_{0}^{\infty} \frac{e^{-\beta_{I} \gamma}\left(\beta_{I} \gamma\right)^{n} \Gamma(\Omega)}{\left(\beta_{I} \gamma+\beta_{J}\right)^{\Omega} \log (2)(1+\gamma) n !} d \gamma
$$

To facilitate the integral calculation, we can perform the following transformation into Fox-H function

$$
\begin{aligned}
& \left(\beta_{I} \gamma+\beta_{J}\right)^{1-\Omega}=\frac{1}{\beta_{J}^{\Omega} \Gamma(\Omega)} H_{1,1}^{1,1}\left(\frac{\beta_{I}}{\beta_{J}} \gamma \mid \begin{array}{c}
(1-\Omega, 1) \\
(0,1)
\end{array}\right) . \\
& \frac{1}{1+\gamma}=H_{1,1}^{1,1}\left(\gamma \mid \begin{array}{c}
(0,1) \\
(0,1)
\end{array}\right) . \quad ; \quad e^{-\beta_{I} \gamma}=H_{0,1}^{1,0}\left(\beta_{I} \gamma \mid \begin{array}{c}
- \\
(0,1)
\end{array}\right) \text {. }
\end{aligned}
$$

Then, we substitute Eq. (21) and Eq. (22) in Eq. (20) and we compute the integral [25]. Hence, we obtain

$$
\begin{aligned}
& \bar{C}_{E}=\frac{1}{\log (2) \Gamma\left(\alpha_{J}\right)} \sum_{n=0}^{\nu-1} \sum_{q=0}^{n}\left(\begin{array}{l}
n \\
q
\end{array}\right) \frac{1}{n ! \beta_{I} \beta_{I}^{q}}
\end{aligned}
$$

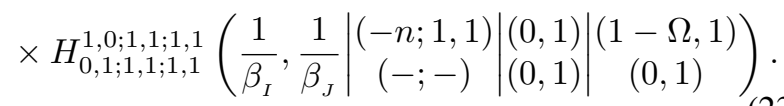

where $\left.H_{m_{1}, n_{1} ; m_{2}, n_{2} ; m_{3}, n_{3}}^{p_{1}, q_{1} ; p_{2}, q_{2} ; p_{3}, q_{3}}(-\mid \cdot)\right)$ is the bivariate Fox $\mathrm{H}$ function [26], [27]. Therefore, by substituting Eq. (19) and Eq. (23) into Eq. (15), we obtain the average secrecy capacity as given on the top of the next page.

\section{Numerical Results AND Discussions}

In this Section, and before investigating the security performance of using the jammer, we are discussing firstly the impact of the shadowing(light shadowing $L_{S}$ and dense shadowing $D_{S}$ ) and blockage (light blockage $L_{B}$ and dense blockage $D_{B}$ ) on the legitimate receiver link by studying the Rician Shadowed as a special case of $\mathcal{D}(\cdot)$. Under the values mentioned in [28], we note that for $\left(L_{B}, L_{S}\right)$, we have an outage probability of 0.02 at $\mathrm{SNR}=20 \mathrm{~dB}$. For the same SNR, the outage probability jumps to more than 0.56 in the case of $\left(L_{B}, D_{S}\right)$. Moreover, during the scenario when the transmission link is subject to $\left(D_{B}, L_{S}\right)$, the outage probability is 0.2 at $\mathrm{SNR}=14 \mathrm{~dB}$. However, to maintain the same level of outage (0.2), the communication link needs to satisfy higher SNR of at least $28 \mathrm{~dB}$ when the shadowing becomes dense $\left(D_{B}, D_{S}\right)$. Therefore, we deduce that the shadowing effect dominates the blockage. In other words, our communication system is sensitive to the shadowing effect much more than the blockage density.

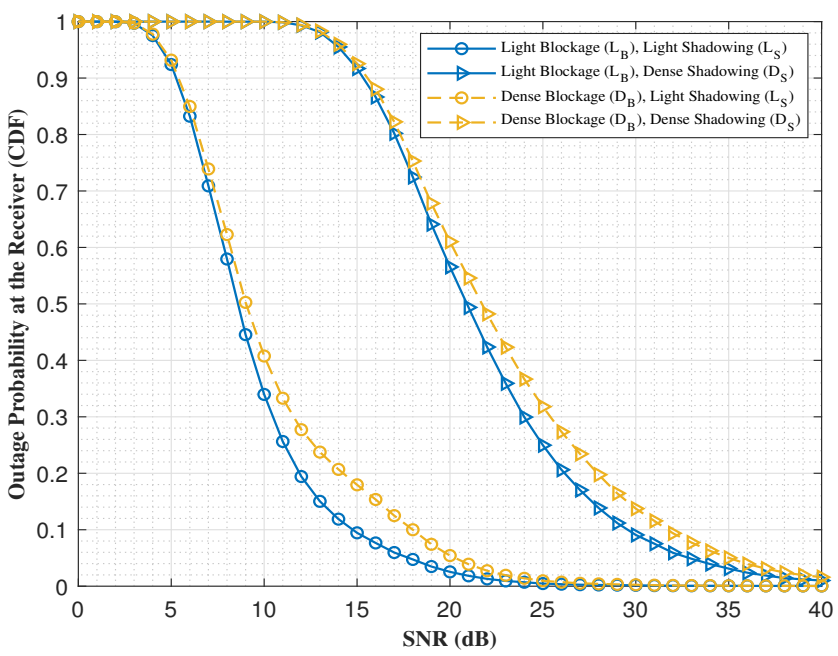

Fig. 2: Impact of the shadowing and the blockage density on the outage probability at the legitimate receiver.

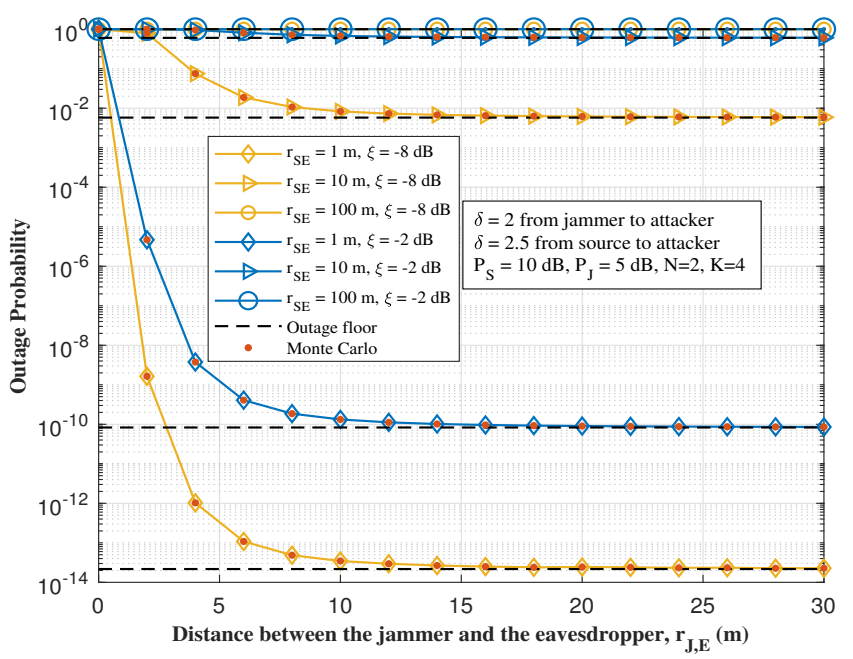

Fig. 3: Outage probability at the eavesdropper versus distance from the jammer to the eavesdropper $r_{J, E}$.

In Fig. 3, we studied the outage probability at $E$ versus a range of $r_{J, E} \in[0 \mathrm{~m} 30 \mathrm{~m}]$, and we fixed $P_{J}$ at $5 \mathrm{~dB}, P_{S}$ $=10 \mathrm{~dB}$. Two different threshold scenarios were proposed: $\zeta$ $=-8 \mathrm{~dB}$ and $\zeta=-2 \mathrm{~dB}$. If we suppose that to ensure secure communication, the outage probability should be more than $10^{-2}$ for $\zeta=-8 \mathrm{~dB}$. In this case, $r_{J, E}$ should be less than $1 \mathrm{~m}$ 


$$
\begin{aligned}
\bar{C}_{s}=\frac{1}{\log (2)}\left\{\frac{\left[\left(s_{R}-1\right) \bar{\gamma}_{L}\right]^{s_{R}} T_{R}^{\mu_{R}}}{B\left(s_{R}, \mu_{R}\right)}\left(\frac{c_{R}}{\left.c_{R}+\mu_{R} \kappa_{I}\right)}\right)^{c_{R}} \sum_{i=0}^{\infty} \frac{\left(c_{R}\right)_{i}\left(s_{R}+\mu_{R}\right)_{i}\left(K_{R} \mu_{R} \kappa_{I}\right)^{i}}{i !\left(\mu_{R}\right)_{i} \Phi^{\eta} \Gamma(\eta)} G_{3,3}^{3,2}\left(\frac{T_{R}}{\Phi} \mid \begin{array}{c}
1-\eta,-\alpha, 1-\alpha \\
0,-\alpha,-\alpha
\end{array}\right)\right. \\
\left.-\frac{1}{\Gamma\left(\alpha_{J}\right)} \sum_{n=0}^{\nu-1} \sum_{q=0}^{n}\left(\begin{array}{c}
n \\
q
\end{array}\right) \frac{1}{n ! \beta_{I} \beta_{I}^{q}} H_{0,1 ; 1,1 ; 1,1}^{1,0 ; 1,1 ; 1,1}\left(\frac{1}{\beta_{I}}, \frac{1}{\beta_{J}}\left|\begin{array}{c}
(-n ; 1,1) \\
(-;-)
\end{array}\right| \begin{array}{c}
(0,1) \mid(1-\Omega, 1) \\
(0,1)
\end{array} \mid \begin{array}{c}
(0,1)
\end{array}\right)\right\}
\end{aligned}
$$

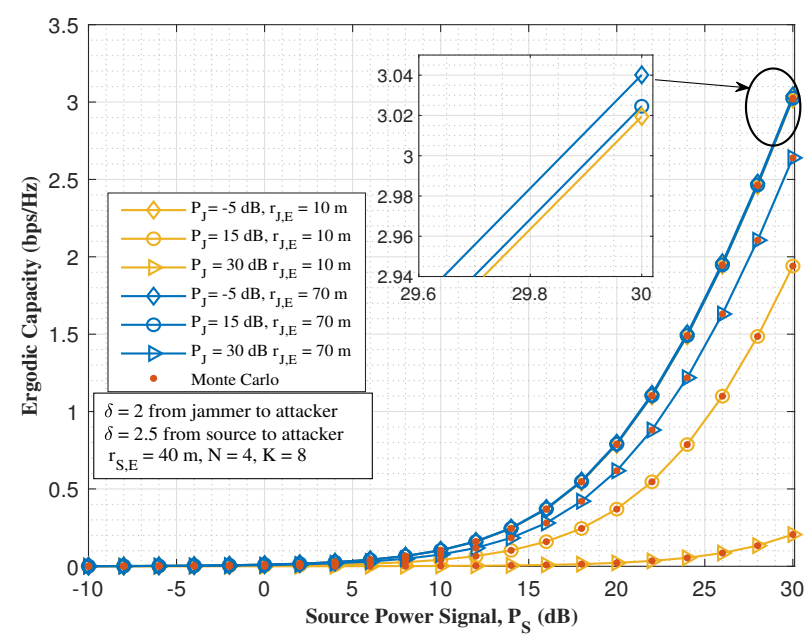

Fig. 4: Effect of the jamming power signal $P_{J}$ on the ergodic capacity at the eavesdropper end.

when the attacker is very close to the source $\left(r_{S, E}=1 \mathrm{~m}\right)$. If the attacker starts to be $10 \mathrm{~m}$ away from $S$, the communication link is protected if $r_{J, E}<15 \mathrm{~m}$. In the second scenario, when the security requirements necessitate $\zeta=-2 \mathrm{~dB}$, the jammer should be at most $1.5 \mathrm{~m}$ away from the eavesdropper to accomplish an outage probability of more than $10^{-2}$ in case that $r_{S, E}=1 \mathrm{~m}$. Both scenarios emphasize on the impact of the distance separating $J$ from $E$ in the critical case where $r_{S, E} \leq 10 \mathrm{~m}$. In those scenarios, the use of the $J$ will be very effective to protect the communication link within a short distance range. As $r_{J, E}$ increases, the outage cannot decrease anymore and we have an outage floor. This is due to the impact of the path loss on the jamming signal, of course under the aforementioned fading model, power setups, and number of antennas at $J$. Hence, to increase the jamming range of $J$ and mitigate the path loss effect, we have to increase $P_{S}$ and $\mathrm{K}$, which will be discussed with further details in the Fig. 5 .

Fig. 4 demonstrates the impact of the jamming power generated by the jammer $P_{J}$ on the ergodic capacity at the eavesdropper $E$ with respect to the source power $P_{S}$. In the first scenario, by fixing $r_{J E}=10 \mathrm{~m}$, we can observe that increasing $P_{J}$ has no effect on the ergodic capacity for low transmission power region $P_{S} \in[-10 \mathrm{~dB}, 8 \mathrm{~dB}]$, since the average capacity is already null because of the low values of $P_{S}$. Hence, using a jammer will not make any difference within this power interval. However, for high $P_{S}$ values such as $25 \mathrm{~dB}$ and by using $P_{J}=-5 \mathrm{~dB}$, the ergodic capacity reaches about $1.7 \mathrm{bps} / \mathrm{Hz}$. Therefore, we need to increase the jamming

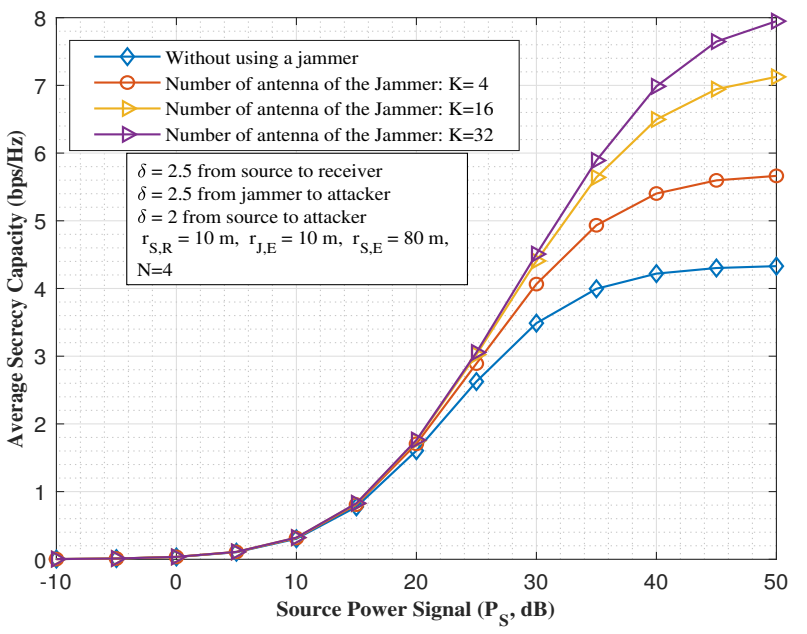

Fig. 5: Average secrecy capacity for different number of antenna at the jammer.

power to at least $P_{J}=15 \mathrm{~dB}$ to decrease the average capacity to $0.95 \mathrm{bps} / \mathrm{Hz}$ and to $P_{J}=30 \mathrm{~dB}$ to achieve a rate $=0.05$ $\mathrm{bps} / \mathrm{Hz}$. In the second scenario, when $r_{J, E}=70 \mathrm{~m}>r_{S, E}$ (the attacker is closer to the BS than to the jammer), we remark that for low range of $P_{S}$, the influence of the AN signal is similar to the previous scenario. On the other hand, for a high $P_{S}$, the difference between the rate corresponding to $P_{J}=-5$ $\mathrm{dB}$ and $P_{J}=15 \mathrm{~dB}$ is negligible, where we need at least 30 $\mathrm{dB}$ at the jammer to decrease the rate from $3.1 \mathrm{bps} / \mathrm{Hz}$ to 2.7 bps/Hz. Therefore, the communication could be secured with low jamming power if $r_{J, E}<r_{S, E}$, otherwise, we need to increase $P_{S}$.

Now, we are focusing of the relation between the study of the average secrecy capacity $\overline{C_{s}}$ and the number of antennas $\mathrm{K}$, at the jammer. As shown if Fig. 5 and by fixing the number of antennas at the BS to $\mathrm{N}=4$, we observe that the average secrecy capacity for different values of $\mathrm{K}$ are tightly close within the BS power $\in[-10 \mathrm{~dB} 18 \mathrm{~dB}]$. We note that as $P_{S}$ increases, we start to notice the impact of $\mathrm{K}$ on the average secrecy capacity. If we need $\overline{C_{s}}$ equal to at least $5.5 \mathrm{bps} / \mathrm{Hz}$ for $P_{S}=35 \mathrm{~dB}$, we can only achieve $4 \mathrm{bps} / \mathrm{Hz}$ when we are not using any jammer. To satisfy such requirement, we need at least 4 antennas. Therefore, the number of antennas K, has a major impact on the performances of security scheme.

\section{CONCLUSION}

In this paper, we examined the PLS in the wireless vehicular network while the communication is subject to an 
eavesdropping attack. We proposed the use of a friendly jammer that will transmit AN to perturb the attacker's channel and decrease its SINR. As a channel model, we adopted the recently proposed Double $\kappa-\mu$ Shadowed Fading model, which provides a variety of fading distribution models. To evaluate the security performance, we studied the outage probability and the secrecy capacity with respect to special fading models such as Rician shadowed and Nakagami-m. The results showed that the jammer has a significant impact on the security performance where we can obtain a notable outage probability at the eavesdropping end, especially for short range distance separating the jammer from the attacker. Moreover, the plots demonstrated the improvement of the average secrecy capacity by equipping the jammer with massive number of antennas.

\section{APPENDIX}

To prove that Nakagami-m distribution is a special case of the envelope of $\mathcal{D}(\cdot)$, we can refer to the envelope equation [20, Eq. (5)] and substitute $c_{l}=s_{l}=\infty, \kappa_{l}=0$ and $\mu_{l}=\mathrm{m}$. Then we perform the following computation:

$$
\begin{aligned}
& \lim _{\substack{c_{l} \rightarrow \infty \\
s_{l} \rightarrow \infty}} f_{X_{l}}(X)=\lim _{\substack{c_{l} \rightarrow \infty \\
s_{l} \rightarrow \infty}} \frac{2\left(s_{l}-1\right)^{s_{l}} c_{l}^{c_{l}} T_{l}^{\mu_{l}} X^{2 \mu_{l}-1} \hat{X}^{2 s_{l}}}{\left(T_{l} X^{2}+\left(s_{l}-1\right) \hat{X}^{2}\right)^{s_{l}+\mu_{l}}} \\
& \frac{\left(c_{l}+\mu_{l} \kappa_{l}\right)^{-c_{l}}}{B\left(s_{l}, \mu_{l}\right)} \times_{2} F_{1}\left(c_{l}, s_{l}+\mu_{l} ; \mu_{l} ; \frac{K_{l} \mu_{l} \kappa_{l} X}{T_{l} X+\left(s_{l}-1\right) \hat{X}^{2}}\right) \\
& =\lim _{s_{l} \rightarrow \infty} 2 m^{m}\left(\frac{s_{l}-1}{\frac{m X^{2}}{\hat{X}^{2}}+s_{l}-1}\right)^{s_{l}} \frac{X^{2 m-1} \Gamma\left(s_{l}+m\right)}{\hat{X}^{2\left(s_{l}+m\right)} \Gamma\left(s_{l}+m\right)} \\
& =\frac{2}{\Gamma(m)}\left(\frac{m}{\hat{X}^{2}}\right)^{m} \exp \left(-\frac{m X^{2}}{\hat{X}^{2}}\right) X^{2 m-1},
\end{aligned}
$$

where $X$ is the random variable, $m$ is the shape parameter, $\hat{X}$ is the root mean square (rms) of the signal envelope, $T_{l}$ $=\mathrm{m}, K_{1}=0,{ }_{2} F_{1}(\cdot, \cdot ; \cdot ; 0)=0$ according to the identity: [24, 07.23.03.0001.01], and $\hat{X}^{2}$ is the controlling spread parameter.

\section{REFERENCES}

[1] W. Tong, A. Hussain, W. X. Bo, and S. Maharjan, "Artificial intelligence for vehicle-to-everything: A survey," IEEE Access, vol. 7, pp. 10823 $10843,2019$.

[2] S. S. Doddalinganavar, P. V. Tergundi, and R. S. Patil, "Survey on deep reinforcement learning protocol in vanet," in 2019 1st International Conference on Advances in Information Technology (ICAIT), 2019, pp. 81-86.

[3] E. Balti and B. K. Johnson, "Tractable approach to mmwaves cellular analysis with fso backhauling under feedback delay and hardware limitations," IEEE Transactions on Wireless Communications, vol. 19 , no. 1, pp. 410-422, 2020 .

[4] E. Balti, M. Guizani, B. Hamdaoui, and B. Khalfi, "Aggregate hardware impairments over mixed rf/fso relaying systems with outdated csi," IEEE Transactions on Communications, vol. 66, no. 3, pp. 1110-1123, 2018.

[5] Y. Yang, Z. Gao, Y. Ma, B. Cao, and D. He, "Machine learning enabling analog beam selection for concurrent transmissions in millimeter-wave $\mathrm{v} 2 \mathrm{v}$ communications," IEEE Transactions on Vehicular Technology, vol. 69, no. 8, pp. 9185-9189, 2020.

[6] E. Balti, M. Guizani, and B. Hamdaoui, "Hybrid rayleigh and doubleweibull over impaired rf/fso system with outdated csi," in 2017 IEEE International Conference on Communications (ICC), 2017, pp. 1-6.

[7] E. Balti, N. Mensi, and S. Yan, "A modified zero-forcing max-power design for hybrid beamforming full-duplex systems," 2020.
[8] A. Hussein, I. H. Elhajj, A. Chehab, and A. Kayssi, "Sdn vanets in 5g: An architecture for resilient security services," in 2017 Fourth International Conference on Software Defined Systems (SDS), 2017, pp. 67-74.

[9] E. Balti, M.Guizani, B. Hamdaoui, and Y. Maalej, "Partial relay selection for hybrid rf/fso systems with hardware impairments," in 2016 IEEE Global Communications Conference (GLOBECOM), 2016, pp. 1-6.

[10] E. Balti, M. Guizani, B. Hamdaoui, and B. Khalfi, "Mixed rf/fso relaying systems with hardware impairments," in GLOBECOM 2017 - 2017 IEEE Global Communications Conference, 2017, pp. 1-6.

[11] A. H. Khosroshahi, P. Keshavarzi, Z. D. KoozehKanani, and J. Sobhi, "Acquiring real time traffic information using vanet and dynamic route guidance," in 2011 IEEE 2nd International Conference on Computing, Control and Industrial Engineering, vol. 1, 2011, pp. 9-13.

[12] N. Mensi, M. Guizani, and A. Makhlouf, "Study of vehicular cloud during traffic congestion," in 2016 4th International Conference on Control Engineering Information Technology (CEIT), 2016, pp. 1-6.

[13] Y. Maalej, A. Abderrahim, M. Guizani, B. Hamdaoui, and E. Balti, "Advanced activity-aware multi-channel operations1609.4 in vanets for vehicular clouds," in 2016 IEEE Global Communications Conference (GLOBECOM), 2016, pp. 1-6.

[14] Deeksha, A. Kumar, and M. Bansal, "A review on vanet security attacks and their countermeasure," in 2017 4th International Conference on Signal Processing, Computing and Control (ISPCC), 2017, pp. 580585.

[15] N. Mensi, A. Makhlouf, and M. Guizani, "Incentives for safe driving in vanet," in 2016 4th International Conference on Control Engineering Information Technology (CEIT), 2016, pp. 1-6.

[16] H. Wang, T. Zheng, and X. Xia, "Secure miso wiretap channels with multiantenna passive eavesdropper: Artificial noise vs. artificial fast fading," IEEE Transactions on Wireless Communications, vol. 14, no. 1, pp. 94-106, 2015

[17] O. Cepheli and G. K. Kurt, "Analysis on the effects of artificial noise on physical layer security," in 2013 21st Signal Processing and Communications Applications Conference (SIU), 2013, pp. 1-4.

[18] V. Bankey and P. K. Upadhyay, "Improving secrecy performance of land mobile satellite systems via a uav friendly jammer," in 2020 IEEE 17th Annual Consumer Communications Networking Conference (CCNC), 2020, pp. 1-4.

[19] B. Li, Y. Zou, J. Zhou, F. Wang, W. Cao, and Y. Yao, "Secrecy outage probability analysis of friendly jammer selection aided multiuser scheduling for wireless networks," IEEE Transactions on Communications, vol. 67, no. 5, pp. 3482-3495, 2019.

[20] N. Simmons, C. R. Nogueira da Silva, S. L. Cotton, P. C. Sofotasios, S. Ki Yoo, and M. D. Yacoub, "The double shadowed $\kappa-\mu$ fading model," in 2019 International Conference on Wireless and Mobile Computing, Networking and Communications (WiMob), 2019, pp. 1-6.

[21] H. Stefanovic, V. Stefanovic, A. Cvetkovic, J. Anastasov, and D. Stefanovic, "Some statistical characteristics of a new shadowed rician fading channel model," in 2008 The Fourth International Conference on Wireless and Mobile Communications, 2008, pp. 235-240.

[22] I. S. Gradshteyn and I. M. Ryzhik, Table of integrals, series, and products, 7th ed. The Netherlands:Academic, 2007.

[23] E. Balti and M. Guizani, "Mixed rf/fso cooperative relaying systems with co-channel interference," IEEE Transactions on Communications, vol. 66, no. 9, pp. 4014-4027, 2018.

[24] W. R. Inc., "Mathematica, Version 12.1," champaign, IL, 2020. [Online]. Available: https://www.wolfram.com/mathematica

[25] P. K. Mittal and K. C. Gupta, "An integral involving generalized function of two variables," Proceedings of the Indian Academy of Sciences Section A, vol. 75, no. 3, pp. 117-123, 1972.

[26] E. Balti and M. Guizani, "Impact of non-linear high-power amplifiers on cooperative relaying systems," IEEE Transactions on Communications, vol. 65 , no. 10, pp. 4163-4175, 2017.

[27] E. Balti, "Analysis of hybrid free space optics and radio frequency cooperative relaying systems," Master's thesis, 2018.

[28] A. Abdi, W. C. Lau, M. . Alouini, and M. Kaveh, "A new simple model for land mobile satellite channels: first- and second-order statistics," IEEE Transactions on Wireless Communications, vol. 2, no. 3, pp. 519$528,2003$. 